\title{
COBAM News
}

\section{Spécial $20^{\text {ème }}$ anniversaire du CIFOR COBAM : Bilan à mi-parcours}

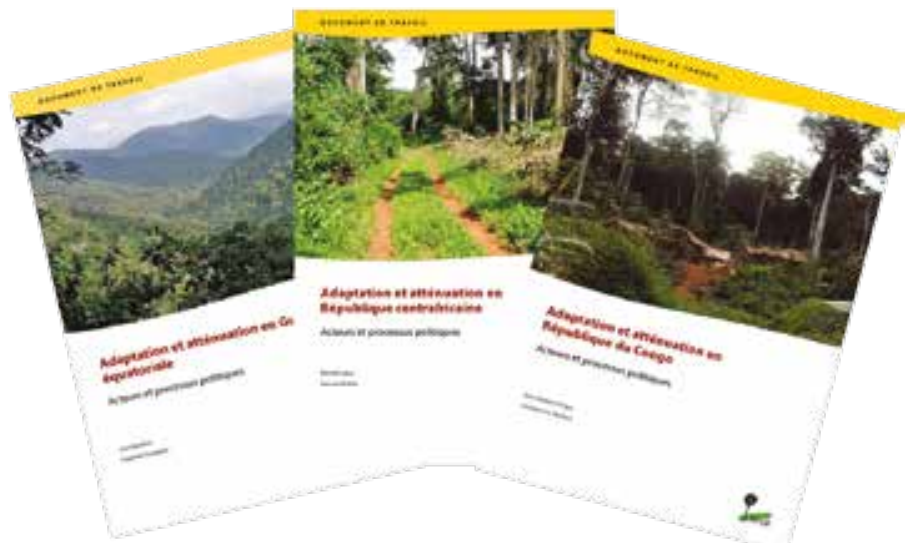

- Quelles sont les actions menées?

- Quels sont les résultats obtenus?

- Quelles sont les perspectives?

Pour répondre à ces questions et à bien d'autres, nous avons préparé ce numéro spécial

\section{Editorial}

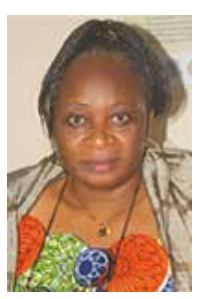

\section{Changement climatique : un cheveu dans la soupe des politiques environnementales en Afrique centrale?}

Anne Marie Tiani

L'Afrique centrale est le théâtre de plusieurs drames, désormais routiniers : à savoir les conflits civils, la pauvreté et les déplacements qui en résultent, l'insécurité aux frontières qui entraine le massacre des éléphants, les enlèvements, le phénomène des coupeurs de route, etc. les Gouvernements sont confrontés au quotidien à toutes sortes de challenges, qui affluent de toutes parts, au niveau de la santé publique (se rappeler l'épidémie de choléra au Cameroun), de la sécurité alimentaire, de l'énergie, des infrastructures, etc...

C'est dans ce contexte que la question du changement climatique doit être analysée. Constitue-t-elle réellement un cheval de bataille pertinent, au milieu des grands défis que doivent affronter les politiques d'Afrique centrale?
La question pourtant se pose, et ce pour plusieurs raisons : les « drames » évoqués plus haut, qui sont autant d'hypothèques au développement de l'Afrique centrale, sont exacerbés par le changement climatique. En effet tout est lié. Prenons l'exemple de la sécurité alimentaire et faisons-en une chaine causale : les perturbations climatiques entraînent des sècheresses ou des inondations, lesquelles provoquent une baisse de rendement et, par ricochet la famine. La part du changement climatique dans chaque désordre structurel peut être ainsi démontrée. Ce ne serait pas aller vite en besogne que de conclure que le changement climatique renforce la pauvreté, alimente les conflits et les déplacements des populations; en d'autres mots, c'est un phénomène qui mérite toute l'attention qui lui est déjà accordée par les Etats, et plus encore.

La question doit plutôt être posée en ces termes : quelle est la valeur ajoutée ou le rôle que joue la question du changement climatique en Afrique centrale ? La question du changement climatique n'est-elle pas fédératrice ou pacificatrice dans notre contexte ? Dans la mesure où les forêts sont une richesse partagée par différents pays, elles peuvent contribuer à renforcer la coopération interétatique. En effet, elles font l'objet d'une politique concertée de gestion, sous la bannière de la COMIFAC, qui se présente comme un niveau stratégique pour les décisions qui engageraient les Etats. De plus, des projets, à l'instar du COBAM, permettent de créer un cadre de discussion qui dépasse les frontières nationales d'une part, et qui établit une passerelle entre les différents niveaux de prise de décision, du local à l'international d'autre part.

Est-ce que la gestion du changement climatique comme d'un problème commun génère des opportunités ? La réponse est positive, on peut tirer plusieurs avantages de la gestion du changement climatique à travers la gestion des forêts, car celles-ci offrent des biens et services qui servent à la fois à l'adaptation au changement climatique et à son atténuation.

Pour conclure, disons que le changement climatique ne doit pas être considéré séparément des autres sources de stress ; à ce titre, il doit faire partie intégrante des politiques de lutte contre la pauvreté du fait qu'il constitue lui-même un des facteurs de pauvreté. La question du changement climatique en Afrique centrale nécessite une approche fédératrice, interétatique et régionale, car les réponses se situent à différents niveaux, local, national, régional et international. C'est un problème commun qui appelle des réponses communes. 


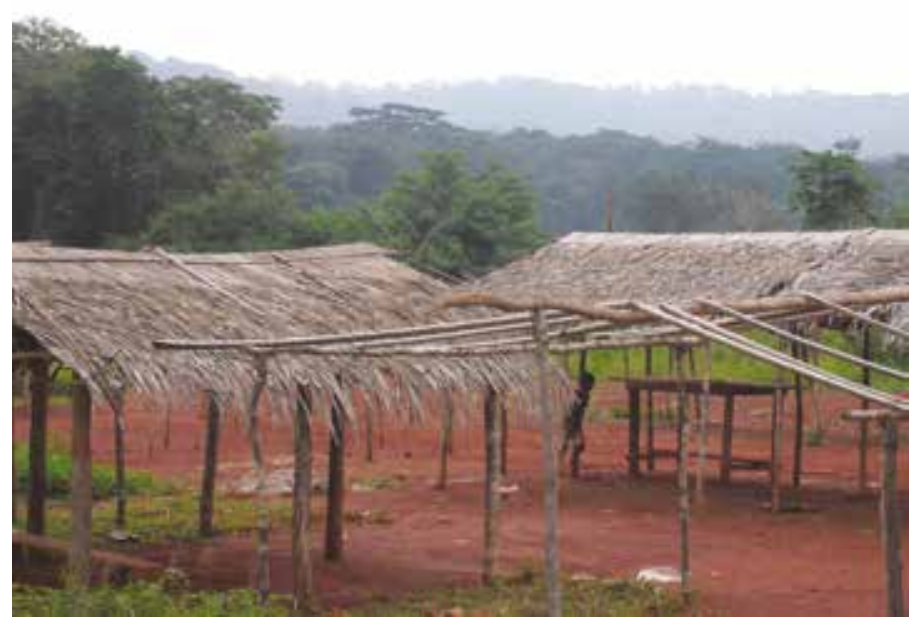

C'est dans cette perspective que COBAM a été initié en 2011. Les premiers résultats de COBAM méritent d'être partagés avec le public. C'est la raison d'être de ce numéro spécial de COBAM news, dans lequel vous trouverez des réponses aux questions suivantes sommes toutes légitimes: «Quelles sont les actions menées depuis son lancement en 2010 ? Quels sont les résultats obtenus?, Quelles sont les perspectives? » Dans ce premier numéro, il sera question de présenter COBAM et ses spécificités, de faire un gros plan sur les paysages dans lesquels les projets pilotes de synergie entre adaptation et atténuation sont mis en œuvre et d'exposer les résultats d'analyse des politiques nationales de changement climatique ou de la vulnérabilité des communautés locales.

\section{COBAM, UN POLE D'EXPERTISE SCIENTIFIQUE ET DE RECHERCHE}

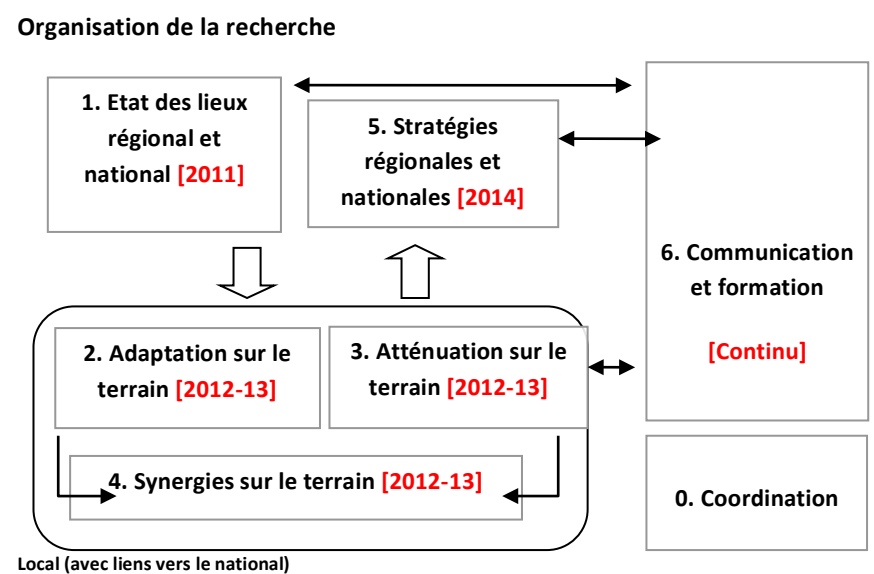

La recherche constitue l'objectif primordial de COBAM, si bien que même les projets pilotes dont COBAM appuie la mise en œuvre sont objets et sujets d'observation.

La recherche porte essentiellement sur l'adaptation et l'atténuation, et sur les possibilités d'en faire une synergie d'acteurs, de politiques et de pratiques, le but étant d'en tirer des leçons pour améliorer les politiques régionales et nationales.
L'atteinte de cette finalité passe par des études intermédiaires. En effet, il faudra entre autres, faire l'état des lieux des politiques nationales et régionales en matière des forêts et de changement climatique, analyser la vulnérabilité des communautés locales et explorer les possibilités d'accroitre leurs capacités d'adaptation, rechercher les causes de la déforestation et de la dégradation des forêts, et explorer les opportunités du REDD à l'échelle régionale et nationale.

Pour en savoir plus : www.cifor/cobam

Et aussi :

COBAM 2011. Changement climatique et forêts dans le Bassin du Congo. Synergies entre l'adaptation et l'atténuation. http://www.cifor.org/cobam/publications.html

\section{Dossier spécial sur le bilan à mi- parcours du projet Changement climatique et forêts dans le Bassin du Congo}

\section{LE PAYSAGE du PACEBCo : ESPACE D’APPLICATION DU PROJET COBAM}

Pourquoi parle-t-on de paysage ? à quoi fait-on référence lorsqu'on parle de paysage du PACEBCo? Ce sont en fait des paysages délimités à cause de leur riche biodiversité, qui nécessitent des actions de conservation. Néanmoins, ils sont aussi sujets à de nombreux pressions et stress, y compris au changement climatique. II s'agit dès lors, pour COBAM, de comprendre comment le changement climatique se superpose à d'autres menaces pour influencer ces paysages de conservation de la biodiversité.

Le Bassin du Congo comporte 12 paysages de conservation jadis délimités dans le programme CARPE. Dans le cadre du Programme d'appui à la conservation des écosystèmes du Bassin du Congo (PACEBCo) financé par la BAD et la CEEAC dont COBAM fait partie, seuls six d'entre eux ont été retenus.

Un paysage de conservation correspond à un vaste territoire non fragmenté, transfrontalier pour la plupart, d'une superficie supérieure à $500 \mathrm{~km}^{2}$ et pouvant abriter des écosystèmes non forestiers comme des lacs ou des fleuves. En Afrique centrale, la spécificité de ces paysages est qu'ils sont des espaces identifiés et gérés pour leur richesse exceptionnelle en biodiversité.

Les six paysages en question sont peu connus du grand public. Qui saurait repérer sur la carte ci-dessus les paysages du PACEBCo suivants: Tri-national de la Sangha; Virunga, lac Télé-Lac Tumba, Maringa-Lopori-Wamba, Maiko-Tayna-Kahuzi-Biega ou Monte AlénMonts de Cristal ? Combien savent que la TNS a été inscrite en 2012 sur la liste du patrimoine mondial de I'UNESCO ? Que l'habitat des gorilles de montagnes dans le Virunga, principal attrait touristique et substantielle source de devises au Rwanda est aujourd'hui menacé par la déforestation et la dégradation des forêts? 


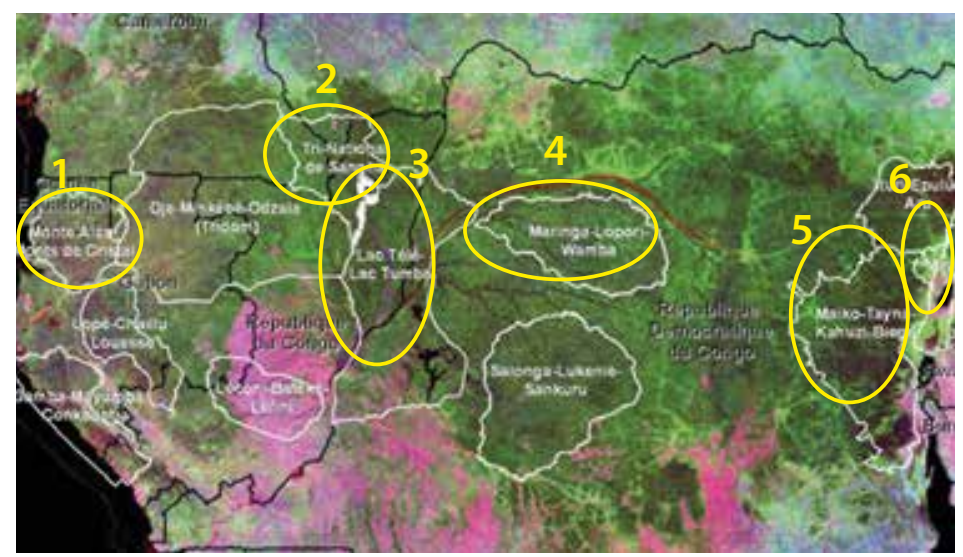

\title{
In vitro
}

EXPLORER LES POSSIBILITES DE SYNERGIE ENTRE LES POLITIQUES NATIONALES D'ADAPTATION ET D'ATTENUATION.

\author{
Eugene Loh Chia
}

Les politiques environnementales et forestières nationales intègrentelles les questions climatiques? Sont-elles propices à une synergie entre l'adaptation et l'atténuation?

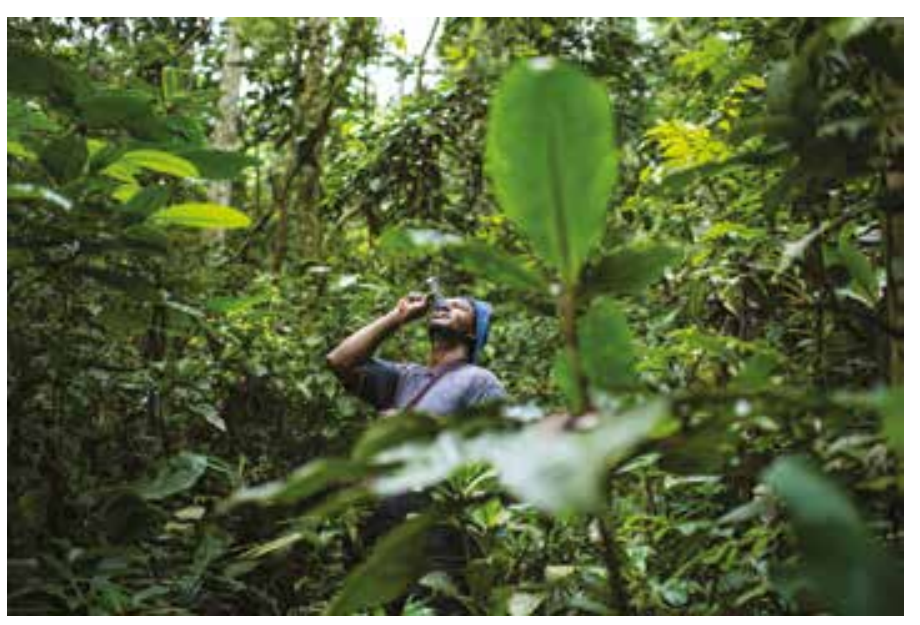

C'est dans ces paysages que COBAM est en train d'accompagner la mise en œuvre de cinq projets expérimentaux de synergie entre l'adaptation et l'atténuation. L'enjeu ici est de trouver comment gérer des espaces où se superposent différentes problématiques souvent conflictuelles. On peut citer entre autres la volonté de la conservation de la biodiversité face à l'urgence du développement dans un contexte d'insécurité, de précarité, de pauvreté généralisée ; la vulnérabilité des populations aux changements climatiques et l'accélération de la déforestation et de la dégradation des écosystèmes forestiers, pourvoyeurs des biens et services et abri pour la biodiversité. Comment mettre en synergie l'adaptation qui est une nécessité, l'atténuation et la conservation de la biodiversité ? C'est à ces questions que COBAM cherche des pistes de réponse dans ces paysages.

Les informations sur les paysages du PACEBCo sont disponibles en Français et en Anglais. Pour plus d'informations, prière de suivre le lien www.cifor/cobam/ pour consulter les documents suivants, disponibles:

Le paysage de la Trinationale de la Sangha. : http://www.cifor.org/ cobam/publications.html

Le paysage de Maringa-Lopori-Wamba. : http://www.cifor.org/ cobam/publications.html

Le paysage de Lac Tele - Lac Tumba. : http://www.cifor.org/cobam/ publications.html

Le paysage de Maiko-Tayna-Kahuzi-Biega. http://www.cifor.org/ cobam/publications.html

Le Paysage Virunga. http://www.cifor.org/cobam/publications.html

Le Paysage Monte Alén/ Monts de Cristal. http://www.cifor.org/ cobam/publications.html

La recherche sur les politiques d'atténuation et d'adaptation menée par COBAM a pour double objectif de comprendre les avancées entrepris par les Etats dans les processus d'adaptation aux changements climatiques et de REDD+, les acteurs impliqués dans les 2 processus et les interactions entre ces différents acteurs.

Cette recherche vise à apporter aux preneurs de décisions nationaux des informations et analyses pouvant les aider à concevoir et à mettre en œuvre les politiques et les projets d'adaptation au changement climatique et de réduction des émissions de carbone dans les forêts, avec des impacts équitables et des co-bénéfices sur la réduction de la pauvreté, l'amélioration des services écosystémiques autres que le carbone, ainsi que la protection des modes d'existence et des droits locaux. Elle examine enfin les possibilités de synergie entre l'adaptation et l'atténuation et analyse les contraintes de la mise en œuvre de la synergie.

Tous les pays du Bassin du Congo sont signataires de conventions internationales ou régionales sur le changement climatique, à l'instar de la Convention-cadre des Nations Unies sur les changements climatiques (CCNUCC), le protocole de Kyoto ou la Commission des forêts d'Afrique centrale (COMIFAC). Toutefois, l'impact de ces engagements sur le processus politique de la lutte contre le changement climatique dans la région est encore mitigé, le contexte étant plutôt caractérisé par un manque de capacités financières, techniques et humaines.

Nous avons mené des recherches sur les processus politiques d'adaptation et d'atténuation du changement climatique dans trois pays du Bassin du Congo : En Guinée équatoriale, le processus politique sur le climat s'avère lent ; le pays est dans la préparation de sa première Communication nationale à la CCNUCC et sa Note d'idée de projets (R-PIN). En République du Congo, des problèmes d'ordre administratif, la dépendance excessive vis-à-vis du savoirfaire externe, le lent décaissement des fonds de partenaires internationaux et le manque d'activités de démonstration sont 
autant d'entraves à la bonne marche du processus. La République centrafricaine, par contre, a déjà soumis son Plan d'adaptation national d'action (PANA). D'autres études sont en cours en RDC et au Cameroun

Notre analyse montre que tous les pays du Bassin du Congo sont engagés dans les processus de REDD+ et d'adaptation, mais à des vitesses différentes, en fonction des intérêts et des supports externes reçus. Elle montre également que les opportunités pour une combinaison entre les politiques d'adaptation et d'atténuation existent et doivent être explorées en profondeur.

Par ailleurs, les pays du Bassin Congo doivent dépasser le cadre d'élaboration des documents comme obligations à des conventions internationales, pour aller vers la conception de véritables plans d'adaptation et d'atténuation rigoureux, basés sur les résultats de recherche de qualité.

\section{Pour en savoir plus:}

- $\quad$ Nguema J. et Pavageau C. 2012 Adaptation et atténuation en Guinée équatoriale: acteurs et processus politiques. Document de travail, CIFOR, Bogor, Indonésie.

http://www.cifor.org/cobam/publications.html

- Pongui B. et Kenfack C. 2012 Adaptation et atténuation en République du Congo : acteurs et processus politiques.

Document de travail. CIFOR, Bogor, Indonésie.

http://www.cifor.org/cobam/publications.html

- Gapia M. et Bele Y. 2012 Adaptation et atténuation en

République centrafricaine : acteurs et processus politiques.

Document de travail. CIFOR, Bogor, Indonésie.

http://www.cifor.org/cobam/publications.html

- $\quad$ Nguema J. et Pavageau C. 2013 Cambio climático y bosques en la cuenca del Congo. Sinergias entre la adaptación y la atenuación.

http://www.cifor.org/cobam/publications.html

\section{CHANGEMENT CLIMATIQUE : COUP DE GRACE pour les POPULATIONS du BASSIN du CONGO ?}

Anne Marie Tiani

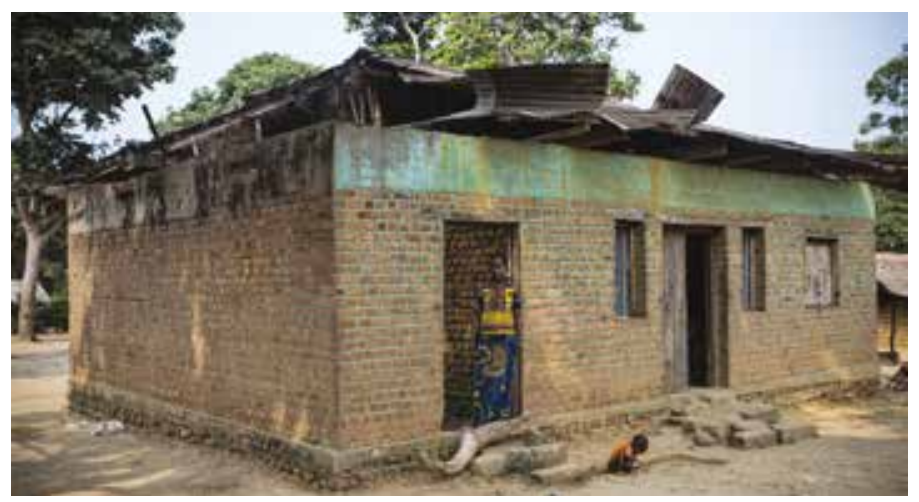

Une étude préliminaire de caractérisation de la vulnérabilité actuelle des communautés locales a été faite dans les paysages et les sites présélectionnés pour la mise en œuvre des projets pilotes de synergie entre adaptation et atténuation. Elle impliquait l'analyse de la vulnérabilité actuelle et la capacité d'adaptation des communautés, afin d'établir les bases pour l'élaboration de scénarios futurs de vulnérabilité et d'identifier les options possibles d'adaptation.

\section{Les populations sont vulnérables face aux multiples stress}

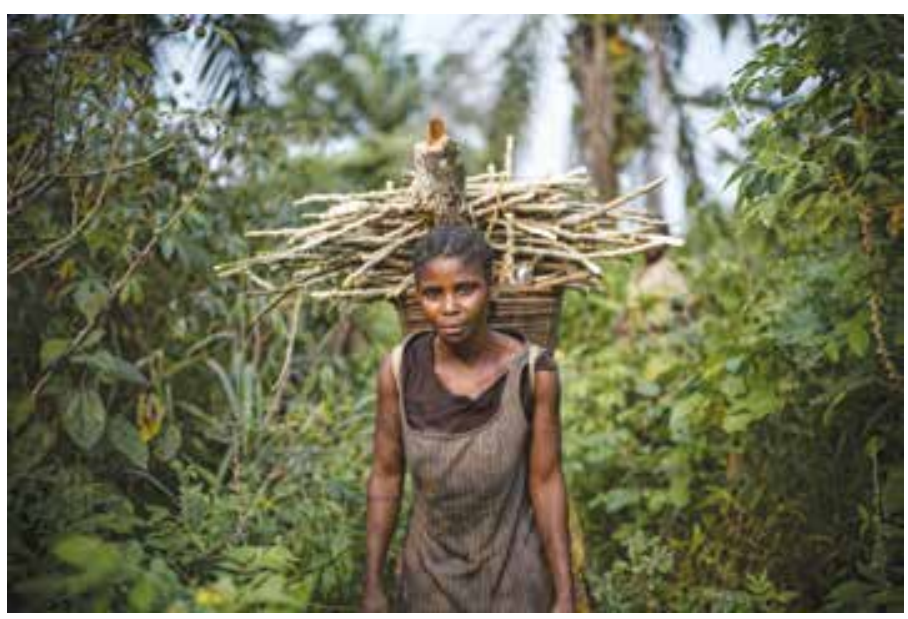

Cette étude réalisée dans cinq sites a montré que les aléas climatiques perçus par les populations sont presque semblables d'un site à l'autre dans le Bassin du Congo : prolongement de la saison sèche, poches de sècheresse en début de saisons pluvieuses, pluies torrentielles et vents violents. Pourtant, les effets de ces aléas sont différenciés en fonction de nombreux facteurs de nature biophysique, sociale ou économique. En effet, cette étude a montré que toutes les couches de la population sont vulnérables à des degrés divers, en fonction de leur histoire d'exposition et à leur sensibilité à d'autres facteurs tels que la position géographique, l'accès aux ressources, les conflits, le niveau de richesse, etc. qui ont façonné leur capacité d'adaptation. Dans tous les sites visités, l'agriculture est l'activité la plus exposée et sensible aux perturbations climatiques telles que la sécheresse et le changement des saisons. L'augmentation des superficies agricoles pour compenser les pertes de rendement a contribué à augmenter la pression sur les forêts et sur les autres ressources naturelles dont dépendent les villages, dessinant ainsi un cycle vicieux qui pourrait accroître la vulnérabilité des communautés locales. Dans la TNS, les enfants et les personnes âgées semblent être les groupes sociaux les plus vulnérables. A Atom en Guinée équatoriale, dans le paysage de Monte Alén- Monts de Cristal, la transition vers une économie de pétrole au cours des deux dernières décennies a entraîné une baisse dans les activités forestières et dans la tendance de la déforestation dans le pays. L'exode rural s'est accéléré, faisant de la disponibilité en main-d'œuvre domestique l'un des facteurs importants de vulnérabilité. Dans le Virunga, les terres des Batwa se situent pour la plupart sur des pentes abruptes ou dans les bas-fonds, les exposant à subir le lessivage des terres et les inondations. A Lukolela dans la province de l'Equateur en RDC, le prolongement de la saison sèche a engendré l'ensablement et l'assèchement des sources et des cours d'eau, la difficulté de navigation et la recrudescence des feux de brousse. Les maladies hydriques, les maladies des plantes et les épidémies chez les bêtes domestiques se sont accentuées et l'épizootie est apparue. Au Bukavu à l'Est de la RDC, on a noté également la recrudescence du paludisme, de la mosaïque de manioc et de la maladie du bétail. Les pluies diluviennes ont entraîné les glissements de terrain, les éboulements et les inondations, occasionnant de pertes importantes dans l'agriculture et des conflits sociaux. 


\section{Face aux perturbations climatiques, la richesse des réactions des communautés locales}

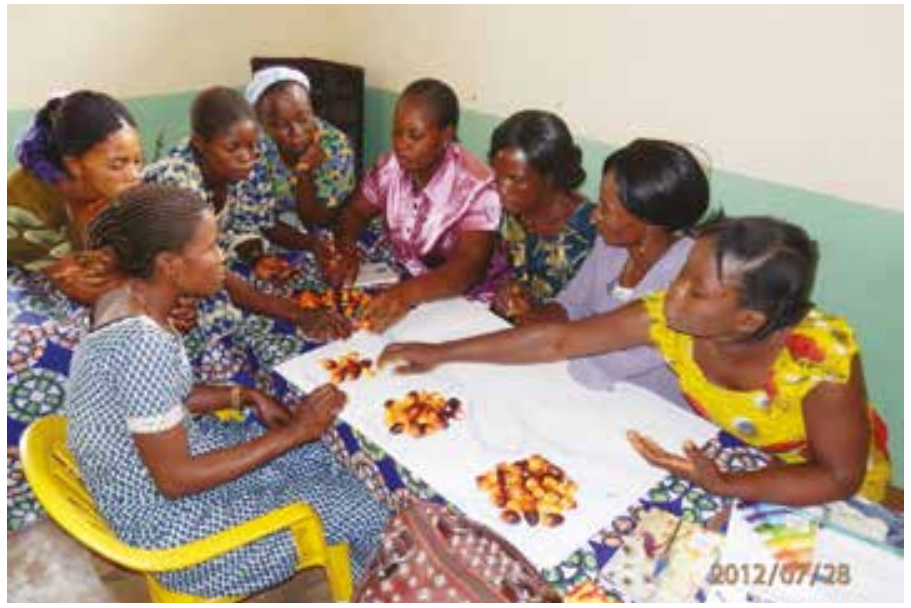

Les perturbations climatiques, coup de grâce pour les communautés locales ? On pourrait être tenté de répondre à cette question par l'affirmative, tant les enjeux sont importants et complexes. Cependant, des marges de manœuvre existent pour les populations locales, et elles ont été explorées dans le cadre de COBAM.

Malgré le fait que l'agriculture pluviale, principale source d'alimentation et de revenus pour les populations locales et les femmes surtout, soit l'activité la plus sensible aux perturbations climatiques, plusieurs types de stratégies d'adaptation ont été recensés.

Dans la zone de forêts riches, la forêt apparaît comme un filet de sécurité parce qu'elle constitue une provision des biens et services alternatifs. Dans la TNS, en dehors de l'agriculture, les populations collectent des PFNL, exercent la chasse et l'exploitation minière artisanale. En outre, les villages sont généralement bien reliés aux marchés où les groupes de ménages s'organisent conjointement pour vendre leurs produits. Au Bukavu, la construction des haies antiérosives, la culture en terrasse, l'usage des gabillons, les tentatives de reboisement, l'exploitation des marais, l'utilisation des foyers améliorés et la recherche des variétés améliorées résistantes à la sécheresse et aux maladies sont autant de stratégies d'adaptation en cours. Au Rwanda, les filets de sécurité pendant les périodes de stress sont développés en dehors de la forêt, à travers des efforts de diversification des activités. La culture en terrasse, la canalisation des eaux courantes, l'amélioration du réseau routier, l'installation de réservoirs d'eau, l'accroissement de la dévolution des pouvoirs à l'échelon local par le transfert de responsabilité aux collectivités locales complété par la redistribution des revenus touristiques du parc aux communautés locales ont contribué au renforcement de la capacité d'adaptation dans la région.

Des activités innovantes pourraient être introduites pour diversifier davantage les économies locales et les sources de revenus tout en conservant les forêts. Les populations de la TNS ont mentionné l'apiculture, l'aquaculture, l'agroforesterie comme autant d'activités possibles qui élargiraient leur assise économique tout en réduisant leur vulnérabilité aux aléas climatiques. A Atom en Guinée équatoriale, I'agroforesterie, l'agriculture verte et le développement de jardins familiaux, le maintien de la fertilité des sols et le contrôle de l'érosion des sols pourront être envisagés. La formation sur les techniques de traitement, stockage et commercialisation des cultures vivrières et des PFNL pourraient accroître leur qualité et les rendre plus concurrentiels sur les marchés urbains. Au Bukavu, les populations proposent, en plus de l'intensification de l'agriculture et du reboisement, la construction des micro-barrages, l'utilisation du biogaz et des panneaux solaires et l'éolienne pour lutter contre la déforestation et s'adapter aux changements climatiques. Malheureusement, dans cette zone, les efforts sont anéantis par des guerres civiles à répétition.

Des actions pilotes, telles que celles entreprises par le projet COBAM, pourraient être un socle pour un apprentissage systémique qui permet la réflexion et le réajustement. Des mécanismes de renforcement de capacités sont nécessaires pour soutenir un tel processus d'apprentissage et pour capitaliser les acquis à plus ou moins long terme.

Pour en savoir plus

Devisscher T., Bharwani S., Tiani A. M., Pavageau C., Kwack N., Taylor R. 2013. Current vulnerability in the Tri-National de la Sangha Landscape, Cameroon. Working paper. CIFOR, Bogor, Indonesia. http://www.cifor.org/cobam/publications.html

Pavageau C., Bele Y, Tiani A.M.2013. Current Vulnerability in the Virunga Landscape, Rwanda. Briefnote. CIFOR, Bogor, Indonesia. http://www.cifor.org/cobam/publications.html

Devisscher T., Bharwani S., Tiani A. M., Pavageau C. 2013. Current vulnerability in the TNS Landscape, Cameroon. Briefnote. CIFOR, Bogor, Indonesia.

http://www.cifor.org/cobam/publications.html

Pavageau C., Morchain D., Coll Besa M. 2013. Current vulnerability in the Monte Alén-Monts de Cristal landscape, Equatorial Guinea. Briefnote. CIFOR, Bogor, Indonesia.

http://www.cifor.org/cobam/publications.html

Bele M.Y., Tiani A.M., Somorin O.A. and Sonwa D.J. 2013. Exploring vulnerability and adaptation needs to climate change of local communities in the humid forest zone of Cameroon. Climatic change. http://www.springer.com/earth+sciences+and+geography/ atmospheric+sciences/journal/10584

\section{IN SITU}

Le recherche-action est une approche qui va au-delà de la recherche purement extractive. Elle suit le cours des dynamiques pour en tirer des leçons en vue d'améliorer des situations. Dans COBAM, il s'agit d'expérimenter in situ des projets d'adaptation ayant le potentiel de stocker le carbone ou des projets REDD+qui présentent des possibilités d'adaptation au changement climatique.

\section{DE LA RECHERCHE ACTION AUX PROJETS PILOTES SUR LE TERRAIN}

\section{Louis Bernard Cheteu}

La recherche action est une méthode de recherche sociale qui met l'accent non seulement sur une meilleure compréhension du problème, mais essaie aussi de contribuer à la résolution du problème social étudié. 
La recherche est un moyen d'action. Les résultats ne sont jamais des réponses définitives, mais d'autres objets de recherche. On capitalise sur les échecs et sur les succès pour réajuster continuellement. German et al. 2012

La recherche action dans COBAM se fait au travers des projets pilotes qui vont être exécutés dans les différents sites. Cinq projets pilotes ont été sélectionnés à la suite d'un appel à projets. Ce sont des projets d'adaptation ayant le potentiel de stocker le carbone ou des projets REDD qui permettent aux populations de s'adapter au changement climatique. La plupart de ces projets sont des projets d'agroforesterie couplés à la gouvernance. Cependant chacun d'eux présente ses spécificités:

Au Rwanda, I'ONG ARECO Nziza fait la promotion de la foresterie à base communautaire pour la conservation de la biodiversité, l'amélioration des conditions de vie et la lutte contre le changement climatique dans la zone du Parc National des Volcans (PNV) dans le paysage Virunga.

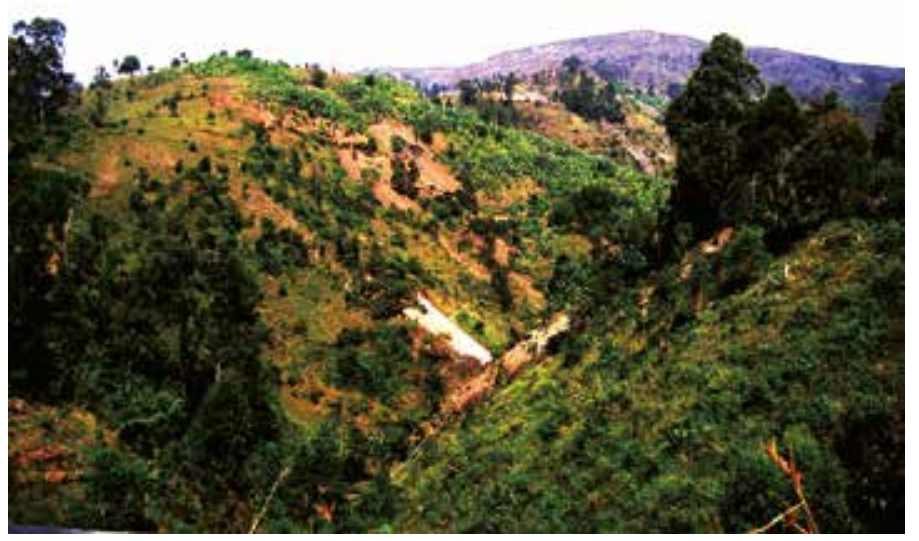

Le projet se propose d'outiller et d'initier les communautés riveraines des forêts aux activités alternatives ou complémentaires génératrices de revenu, afin d'amoindrir la pression qu'elles exercent sur les forêts. Les différentes activités définies par le projet portent sur la restauration des paysages et la prévention de l'érosion des sols; le renforcement des capacités des populations locales pour une gestion productive et durable des ressources naturelles et la création d'activités génératrices de revenu pour les communautés. Les bénéficiaires directs du projet seront particulièrement les membres des coopératives et associations impliquées dans la foresterie et dans la valorisation des filières liées aux PFNL.

En Guinée équatoriale, INDEFOR se propose de supporter la gestion durable des forêts communautaires pour l'adaptation et l'atténuation des effets du changement climatique du paysage Monte Alén-Monts de cristal. II s'agit de réduire la vulnérabilité des populations locales et de contribuer à la réduction de la déforestation et de la dégradation des forêts naturelles du paysage ; ceci, à travers des activités visant à renforcer la capacité des acteurs locaux du paysage sur les concepts clés des changements climatiques et de REDD+, à analyser la vulnérabilité des communautés locales du paysage afin de définir et mettre en œuvre des stratégies d'adaptation et d'atténuation dans les sites sélectionnés.

Le projet COBAM-Lukolela initié en RDC par le Réseau africain de Forêts Modèles (RAFM) vise la mise en œuvre, de manière participative, d'activités qui renforceront les capacités d'adaptation des communautés locales face aux effets du changement climatiques, avec des co-bénéfices en matière de fixation de carbone. Pour cela, le projet entend procéder au reboisement de sept mosaïques dégradées du paysage de la cité de Lukolela ainsi que son périmètre immédiat jusqu'à $15 \mathrm{~km}$ autour des limites de la cité. De plus, les activités telles que la réhabilitation des zones ripériennes et des frayères, la lutte antiérosive sur les berges du fleuve Congo, le développement de l'agrobusiness seront soutenues et des partenariats mobilisés pour leur mise en œuvre.

Dans la TNS, le réseau des ONG du Sud-Est (ROSE) initie un projet de synergie entre l'adaptation et l'atténuation sur le socle de deux forêts communautaires, DJANKORA et MPEMOG. Le projet va évaluer l'application des plans simples de gestion existant et en proposer une révision qui tienne compte des facteurs de vulnérabilité des communautés locales en général et des Baka en particulier. En plus, le projet prévoit de promouvoir des activités génératrices de revenus compatibles avec la conservation des ressources naturelles. L'agriculture sans brulis, l'agroforesterie et la collecte durable des PFNL en font partie.

L'Union pour l'émancipation de la femme autochtone (UEFA) propose une réponse à la vulnérabilité des écosystèmes adjacents du Parc National de Kahuzi-Biega (PNKB) en République démocratique du Congo. Le projet initié par cette ONG vise à améliorer et promouvoir les foyers améliorés, les pratiques agricoles et agro-forestières responsables pour lutter contre la déforestation et la dégradation, liées à la pratique d'agriculture itinérante et à la recherche du bois énergie. II veut également promouvoir les activités génératrices de revenus alternatives en vue de diminuer la pression sur le parc, réduire la pauvreté dans la zone du projet et structurer les communautés pour une bonne gestion des ressources naturelles.

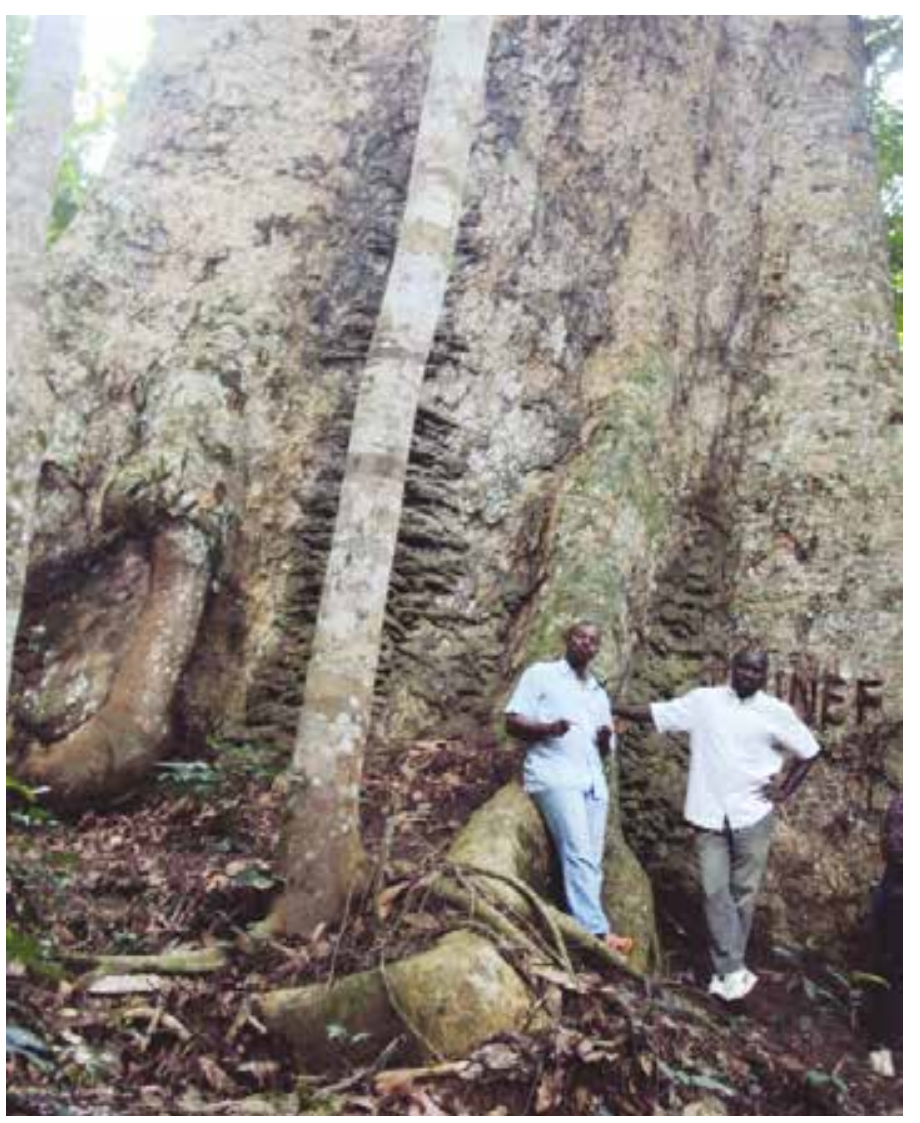


Pour en savoir plus sur la recherche action :

German L. A., Tiani A.-M., Daoudi A., Maravanyika, Tendayi M., Chuma E. 2012. Application of participatory action research to climate change adaptation in Africa: a reference guide International. Development Research Centre and Center for International Forestry Research. http://www.cifor.org/cobam/publications.html

German L. A., Tiani A.-M., Daoudi A., Maravanyika, Tendayi M., Chuma E. 2012. Application de la recherche action participative à l'adaptation aux changements climatiques en Afrique : Guide de référence. Centre de recherche pour le développement international, Centre pour la recherche forestière internationale. http://www.cifor.org/cobam/publications.htm

\section{IN LIBRO}

\section{LA COMMUNICATION dans COBAM MOBILISE tous les VECTEURS MODERNES de TRANSMISSION de MESSAGES}

\section{Merline Touko}

La communication est l'un des objectifs spécifiques du projet COBAM, clairement défini dans le document de projet. COBAM doit « communiquer, partager les connaissances et renforcer les capacités aux échelles locales, sous-nationales et nationales».

La communication a plusieurs effets, qu'on pourrait regrouper en deux pôles principaux : d'une part, elle participe du rayonnement et de la visibilité du projet, et d'autre part, elle veille à la dissémination des résultats sur la REDD+ et adaptation, pour les mettre à la disposition du public.

La communication dans COBAM cible différents acteurs. D'abord les preneurs de décision aux niveaux régional, national et local afin de les éclairer dans leur prise de décision ; ensuite les communautés locales afin de leur donner les outils pour une meilleure participation à la prise de décision ; et enfin la communauté scientifique dans le but de rendre disponible sa contribution à la construction du savoir dans le domaine. Elle adopte, pour cela, plusieurs canaux de transmission, en fonction des cibles visées et des objectifs déjà définis.

Les stratégies de communication de COBAM s'articulent, autour de 3 points essentiels.

\section{L'animation du dialogue science-politique}

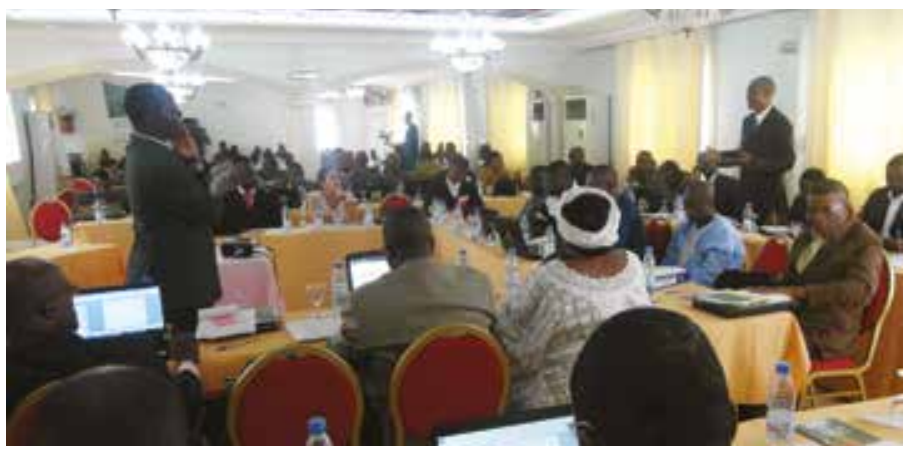

Le CIFOR a contribué à animer et à alimenter le dialogue science politique en Afrique à travers la co-organisation de plusieurs ateliers d'échange, dont celui du lancement du projet à Douala du 12 au 14 mai 2011 ou de la journée scientifique 'Forest Day Central Africa, en marge du Sommet des trois Bassins à Brazzaville en mai 2011; On peut citer également la participation active à de nombreux fora internationaux dont la COP 17 à Durban en 2011, le symposium organisé par IUFRO/FORNESSA à Nairobi en juin 2012, avec l'organisation d'un panel sur le changement climatique dans le Bassin du Congo et sept autres présentations. Plus récemment, on compte l'organisation d'un stand à la Conférence africaine des forêts modèles à Yaoundé en mars 2013.

COBAM a eu une présence remarquée dans l'espace COMIFAC du Sommet de la Terre à Rio du 17 au 23 juin 2012 grâce à plusieurs activités, notamment l'exposition de 6 posters présentant les réalités du Bassin du Congo en matière de changement climatique, la distribution de quatre flyers, en français, anglais et portugais, une présentation dans le panel sur le genre et la foresterie ainsi qu'une interview télévisée avec Pavilion TV. Au total, une cinquantaine présentations et contributions aux débats aux niveaux national, régional et international depuis le lancement du projet.

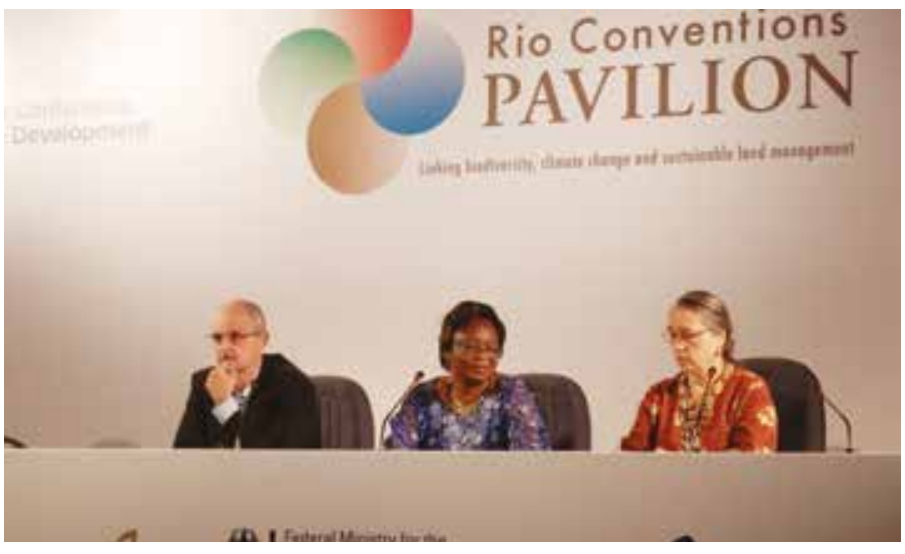

\section{Le renforcement des capacités des décideurs et des populations locales}

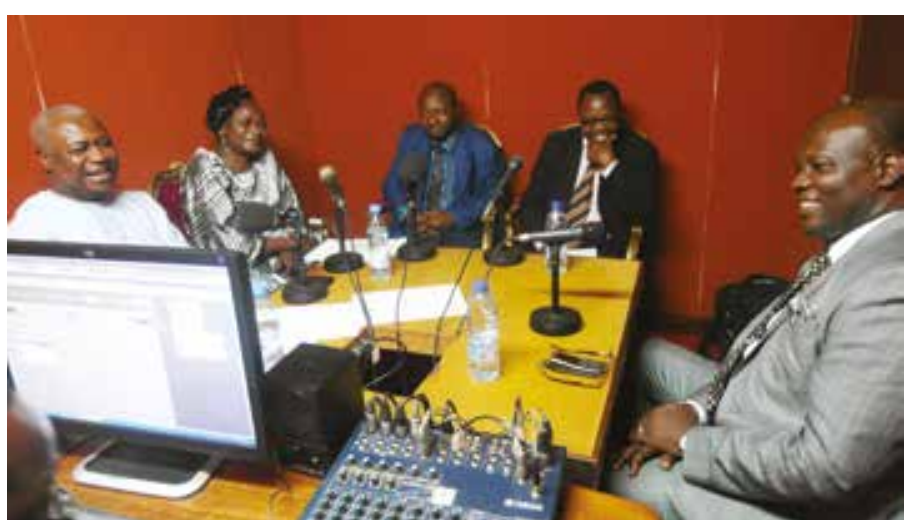

Le renforcement des capacités des décideurs et des populations locales est l'une des missions de COBAM. Plusieurs ateliers de formation ont été organisés en direction des acteurs du changement climatique, parmi lesquels la formation pour la rédaction des projets sur l'adaptation et l'atténuation; I'atelier de formation sur les MRV-REDD+ et les paysages de la biodiversité dans le Bassin du Congo et l'atelier sur la recherche participative (RAP) appliquée à l'adaptation aux changements climatiques. 
De nombreuses publications en cours procèdent également au renforcement des capacités. Elles concernent les concepts relatifs aux changements climatiques. Les premiers résultats ont déjà été obtenus, notamment sur les politiques et les acteurs nationaux, et sur la vulnérabilité et les capacités d'adaptation des communautés locales.

La confection continue d'une liste de diffusion et son utilisation pour la diffusion des informations. Rentre également dans la stratégie de communication la création d'une base de données de plus de 2000 contacts stratégiques vers lesquels sont régulièrement diffusées les informations issues du projet COBAM.

Des média de masse sont mises à contribution, parmi lesquels des documentaires et des émissions radio ou télévisées. En témoigne l'exposition médiatique notoire que constitue l'émission radio mensuelle «Au rythme des saisons », animée Alain Belibi, journaliste émérite de la Radiotélévision camerounaise CRTV. Elle sera bientôt relayée dans les antennes à vocation communautaire comme le Community Radio Network (Rerac) porté par I'IUCN, Radio Okapi en $\mathrm{RDC}$ et par d'autres chaines radio de la région. Elle est néanmoins disponible dans les réseaux sociaux tels que Youtube, et Facebook, ainsi que dans le site web du CIFOR/ COBAM (http://www.cifor.org/ cobam/multimedia.radio.html)

Par ailleurs, depuis novembre 2011, le COBAM dispose d'un site web régulièrement mis à jour : cifor.org /cobam.
Enfin le COBAM communique à travers la production, la diffusion et la distribution de plusieurs autres outils tels que les documentaires, briefs, posters, flyers, et par des gadgets (clés USB, chemises cartonnées, sacs, stylos, auto collants).

\section{La production de l'information sur la vulnérabilité au changement climatique et sur les causes de la déforestation et dégradation dans la région}

COBAM est une unité de recherche du CIFOR, qui a déjà produit plusieurs supports contenant les résultats des recherches sur la vulnérabilité des populations dans les différents sites et sur les causes de la déforestation et de la dégradation des forêts du Bassin du Congo. (Voir plus haut In vitro, In situ)

Pour en savoir plus sur les concepts-clés relatifs au changement climatique, prière de lire les documents suivants

- Locatelli B. 2011. Les synergies entre adaptation et atténuation en quelques mots. Support distribué à travers la liste de distribution du COBAM à plus de 1000 individus dans le Bassin du Congo et ailleurs en Afrique.

http://www.cifor.org/cobam/publications.html

- $\quad$ Pramova E., Locatelli B., Djoudi H. et. Somorin O. A. 2012. Le rôle des forêts et des arbres dans l'adaptation sociale à la variabilité et au changement climatiques. CIFOR, Bogor, Indonésie. http://www.cifor.org/cobam/publications.html

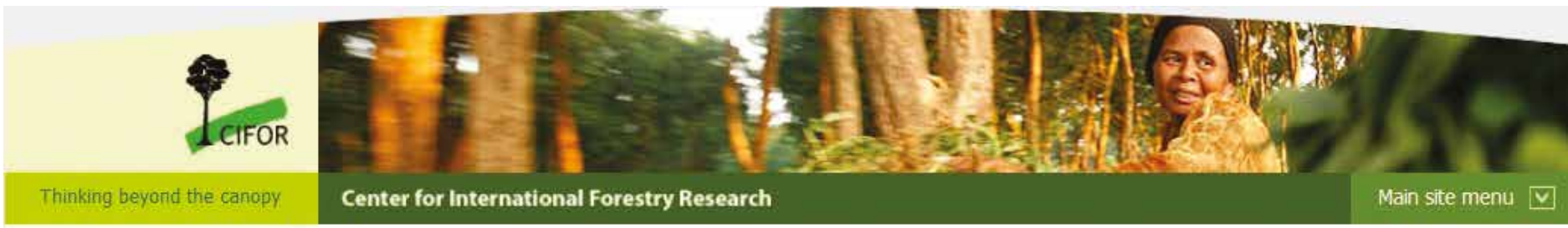

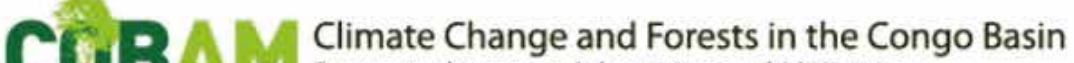
Synergies between Adaptation and Mitigation

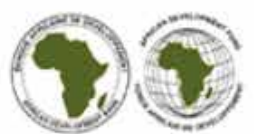

AFRICANDEVELOPMENT BANK GROUP

PEnglish Français
Home

Background

Project

Updates

Publications

Multimedia

Contact

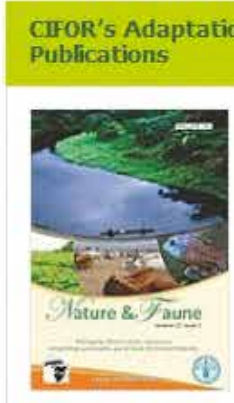

Lien entre forêt, eau et populations f. $1.9 \mathrm{MB}$

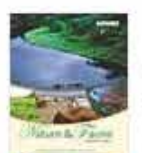

The link between forest, water and people: an agenda to promote in 1 78 क the context

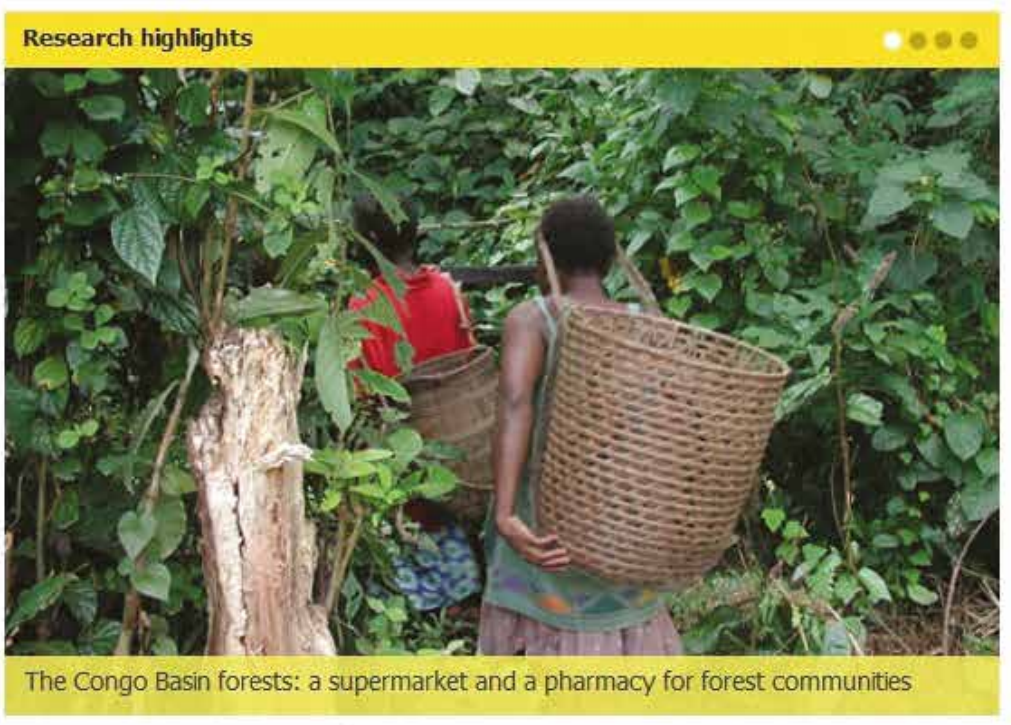

\section{News from WEADAPT More}

Articles

In Ecosystems, Climate Change, Vulnerability and Economics connect - 27 February 2013

- Proceedings of the International Network on Climate Change (INCA) 2012 - 11 February 2013

=11 Building Urban Climate Change Resilience in Bangkok - 23 December 2012

Adaptation layer case studies

* Climate Change Adaptation in Tanzania's Coastal Villages - 24 January 2013

E Participatory 3D modeling for community-based climate change adaptation in BoeBoe community, Choiseul, Solomon Islands, - 14 January 2013

- Climate Adaptation Decision explore

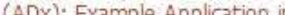




\section{COMPRENDRE LE DIALOGUE SCIENCE- POLITIQUE}

Interview de Denis Sonwa
Cobam News : Que désigne-t-on par dialogue Science-politique? C'est un espace d'échange itératif qui permet aux scientifiques et aux preneurs de décisions de se soutenir mutuellement, et d'identifier ensemble les axes de recherches qui leur semblent prioritaires. Dans un contexte de changement climatique, où l'on doit faire face chaque jour à une masse de données importantes, les scientifiques doivent pouvoir partager les informations actualisées avec les politiques pour qu'ils puissent mieux orienter leurs prises de décisions et les politiques; et à leur tour ceux-ci doivent orienter les scientifiques vers des axes qui méritent des investigations particulières, pour le bien de l'environnement et des populations.

Cobam News : Que fait le COBAM pour promouvoir le dialogue science-politique à tous les niveaux?

Le lien entre les politiques et les scientifiques est mis en exergue: d'abord au niveau de la conception même du projet. Dès la genèse de COBAM, il y a eu plusieurs dialogues entre la structure de recherche qu'est le CIFOR et les structures de prise de décisions, ici la CEEAC et les partenaires comme la COMIFAC et la BAD. C'est de cette communion qu'est né le projet COBAM.

Ensuite au niveau de l'exécution du projet: si vous prenez les études sur la vulnérabilité des populations conduites sur le terrain par COBAM, vous vous rendrez compte qu'à chaque fois qu'on arrivait dans un paysage, on réunissait autour de la même table les acteurs politiques et quelques scientifiques. Ceci dans le but de renforcer le dialogue science-politique.

Et enfin, on l'a aussi vu lors de l'atelier sur les MRV (mesure notification et vérification des stocks de carbones) organisé à Douala par COBAM. Les gestionnaires de paysages, les points focaux changement climatique des paysages, les responsables de la biodiversité des pays, les chercheurs du CIFOR, des structures et des universités ont, durant quelques jours, échangé des informations et emmagasiné des connaissances.

Cobam News : Avez-vous l'impression que les politiques d'Afrique centrale tiennent compte de la science dans le processus de prise de décision?

Ceux qui prennent des décisions le font parfois en tenant compte des informations qu'ils ont reçues lors de leurs cursus académiques. II faut rappeler ici que les manuels sont produits par les scientifiques. Ce qui confère à ces preneurs de décisions un certain background technique. Mais dans un contexte où il y a des découvertes permanentes, il se pose un défi de recyclage. COBAM est donc une possibilité pour les chercheurs de mettre à la disponibilité des politiques les informations qu'ils ont.

De manière globale, les décisions sont prises en tenant compte de ce que les scientifiques ont produit par le passé. Le défi pour les pays du Sud, c'est de pouvoir internaliser les découvertes dans les prises de décisions locales. Avec les projets pilotes de COBAM nous essayons de combler ce vide.

Propos recueillis par Merline Touko

\section{Blogsbuster - La vie de nos blogueurs}

\section{DES POSTS DANS UN BLOG : POUR UNE AUDIENCE ELARGIE ?}

\author{
Eric Essono Tsimi*
}

La révolution numérique a pu désigner, dans la bouche des politiciens, la croissance rapide des technologies de l'information et de la communication et l'innovation dans les systèmes numériques. Parmi les barrières qu'Internet a fait s'écrouler, la circulation des idées est sans doute la plus remarquable. Les acteurs scientifiques se sont appropriés les nouvelles technologies, se sont ouverts au monde et ont fait de la communication un levier de vulgarisation scientifique et d'autopromotion.

Ils ne sont plus ces êtres en apesanteur, solitaires, enfermés dans des cages de Faraday, d'où ils ne sortiraient que pour tenir de hautes conférences. Ils ont pris d'assaut les outils numériques tels que les réseaux sociaux et les blogs. Tout ce qu'il leur a fallu pour cela, c'est du temps. L'argent n'est pas un problème, la création d'un blog sur internet est gratuite, même quand celui-ci est hébergé par des organismes importants, il ne comporte pas comme dans les revues à comité de lecture, de grandes contraintes intellectuelles et matérielles.

Le blog du CIFOR est un espace de liberté où les contributeurs peuvent exprimer des opinions qui se démarquent de la position officielle de leur employeur, des opinions qui, en tout cas, n'ont pas vocation à traduire les vues du CGIAR ou de ses différents centres de recherche. Le mieux cependant étant toujours de s'exprimer pour amplifier, approfondir, ou vulgariser des résultats qui ont fait autorité. Car il s'agit moins d'un blog personnel que d'un blog thématique (blog forests) créé et édité par le CIFOR, non pas par les blogueurs eux-mêmes.

De quoi parle-t-on dans le «forests blog »?

En mars 2013, très récemment donc, Denis Sonwa, chercheur au CIFOR, postait un article initialement paru dans une newsletter d'anciens étudiants de Berkeley: Des difficultés sont en vue si forêts et adaptation ne sont pas prises en compte après Rio +20. C'est que le sujet était d'une pertinence que n'avait pas démentie l'actualité. « II serait illusoire, écrit-il, de penser que nous allons utiliser les forêts pour l'atténuation sans tenir compte de la gestion des effets négatifs du changement climatique sur les peuplements forestiers et sur les communautés. »

Le ton est un tantinet alarmiste, comme dans l'article, toujours en libre accès, de Babatope Akinwande, paru en novembre 2012, où il a pu estimer que les forêts du Bassin du Congo est « sous la menace », car « la pression de déforestation sur [ces] forêts (...) grandit ». Mais la prose des contributeurs peut aussi être juste descriptive comme dans la réflexion du même auteur, intitulée Les communautés forestières au Cameroun ne peuvent pas s'adapter seules au changement climatique, qui est le fruit d'une recherche menée dans le cadre du projet CoFCCA (Forêts du Bassin du Congo et adaptation au changement climatique). 
En tant que lieu d'expression aux fonctionnalités souples, un blog ne vise pas à la seule efficacité ou l'atteinte d'une cible, il est aussi le moyen pour ceux qui y publient des articles de faire valoir leurs idées, de diffuser, sous une forme et un format accessibles, des retours d'expérience ou des recherches menées par ailleurs. Pour une audience élargie donc, mais surtout pour une parole libérée des « carcans » technico-scientifiques (notes infrapaginales, annexes lourdes, bibliographies kilométriques, etc.)

Toutefois, la rigueur, que l'on veuille bien me passer la répétition, reste de rigueur, dans la mesure où tous les articles sont documentés par des hyperliens et des références précises qui assertent, confortent ou développent les thèses des auteurs. Lesquels y gagnent, in fine, un surplus de visibilité et, parfois, de notoriété.

*Journaliste consultant, COBAM

\section{COBAM et les autres...}

\section{Interview de Anne-Marie Tiani}

Cobam News : Dans la constellation des organismes, organisations, projets et centres de recherche, quelle est la spécificité et la pertinence du Projet Cobam?

AMT : COBAM est à la fois un projet de recherche et de recherche action. Dans ces conditions, les résultats de la recherche trouvent directement leur champ d'expérimentation dans des projets pilotes et les leçons tirées de ces expérimentations permettent de réajuster directement le tir. Ceci est d'autant plus important que généralement, de nombreux résultats de recherche sont vite oubliés, faute de trouver des champs pour leur application.

COBAM allie la recherche sur la vulnérabilité avec celle sur les causes de la déforestation et de la dégradation des forêts. Ces deux thématiques sont en général séparées et cette séparation ne permet pas d'explorer à fond les liens causaux, les synergies et les conflits qui peuvent exister entre les deux.

COBAM étudie les possibilités de mettre en synergie l'adaptation et l'atténuation dans des paysages de conservation de la biodiversité. Ce faisant, l'occasion est saisie d'allier la Convention sur la diversité biologique à la Convention-cadre des Nations Unies sur les changements climatiques (CCNUCC). L'approche éco systémique permet de rapprocher des problématiques traitées séparément, et qui ont pourtant une unicité d'acteurs et d'espace.

Par ailleurs, COBAM offre une opportunité quasi unique de faire des recherches couvrant l'ensemble du Bassin du Congo, sur un continuum forêts riches à faible densité de population à l'Ouest, en Guinée équatoriale, à forêts complètement dégradées à forte densité de population à L'Est de la RDC et au Rwanda. Entre les deux, toutes les variantes peuvent être trouvées.

Enfin, COBAM est à la fois inter-scalaire et horizontale : elle va de l'étude des politiques au niveau régional et national à l'analyse de la vulnérabilité et des causes de la déforestation au niveau des ménages. Pour chaque niveau d'intervention, le projet couvre un éventail assez large de cas. C'est ainsi que les études au niveau national couvrent 6 pays et celles au niveau local concernent cinq sites disséminés dans le Bassin du Congo.

Cobam News : Concernant les objectifs, les cibles, les partenaires, y a-t-il eu, en cours de dispositif, des évolutions qui n'étaient pas prévues telles quelles à l'origine? En d'autres termes, le contenu du projet est-il adaptable aux données contextuelles ?

AMT : A ses débuts, COBAM était avant tout un projet d'adaptation. Il se situait en droite ligne du projet CoFCCA, un autre projet d'adaptation initié par le CIFOR entre 2008 et 2010, et coordonné par Dr Sonwa. COBAM se présentait donc comme une confirmation et extension de CoFCCA. Les orientations de COBAM ont évolué pour plusieurs raisons, dont le choix clairement exprimé des politiques régionales pour la REDD+. Il fallait un recentrage, qui a permis d'explorer les possibilités et potentialités de synergies entre l'adaptation et l'atténuation, et ce, en conformité avec le programme de recherche du CIFOR. Ce recentrage était très pertinent puisqu'il a permis d'allier la nécessité d'une recherche sur I'adaptation et l'opportunité d'une autre sur l'atténuation qu'offrait la présence de ce vaste massif forestier, le deuxième au monde.

Cobam News : Y a-t-il des problèmes dont on peut déjà supposer qu'ils ne seront pas résolus à la fin du projet? Existe-t-il un système d'audit ou de suivi évaluation pour anticiper sur ces problèmes? AMT : COBAM est un projet, et en tant que tel, a une durée de vie limitée. De nombreux produits de recherche ne seront disponibles qu'après la durée du projet. C'est toujours ainsi.

Cependant, il est à craindre que les projets pilotes, I'un des résultats-phares du projet, dont on était en droit d'attendre beaucoup surtout en termes de leçons d'expérience, ne puissent pas livrer ses secrets à cause du retard accusé dans leur mise en œuvre.

Il est néanmoins possible d'anticiper sur les résultats par la mise sur pied, en même temps que le projet, d'un système de suivi-évaluation participatif et autocorrectif. Mais pour cela, il faut également disposer de temps nécessaire et des moyens conséquents. Heureusement, la BAD, la CEEAC et le PACEBCo en sont conscients, et nous leur en sommes très reconnaissants.

Propos recueillis par Eric Essono Tsimi 


\section{BREVES}

- Le Bassin du Congo et la forêt amazonienne sont suivis par satellite. Le journal français Le Monde, du 12 avril 2013, indique que le «Sud veut sa part de données satellitaires pour gérer ses ressources » : grâce à un financement de l'AFD, Astrium met déjà à la disposition de six pays du Bassin du Congo les données dont elle dispose. La protection des forêts tropicales ne peut s'envisager aujourd'hui sans le recours aux observations satellitaires. Celles-ci permettraient de réduire la dépendance des pays du Bassin du Congo aux programmes de la coopération internationale.

- Le palmier à huile menace l'écosystème du Bassin du Congo. Selon un récent rapport de la RFUK, plus de 400000 hectares de forêt tropicale du Bassin du Congo sont exploités par les producteurs d'huile de palme. Et même jusqu'à 115 millions d'hectares pourraient l'être, d'où le désir d'expansion de ces sociétés.

- Dans une tribune parue dans le HuffingtonPost, Achim Steiner, Secrétaire général adjoint et Directeur exécutif du Programme des Nations Unies pour l'environnement et le Dr Andrew Steer, Président et Chef de direction de I'Institut des ressources mondiales, estiment qu'une révolution technologique, notamment le Global Forest Watch 2.0 qui sera lancé en 2013, pourra freiner l'exploitation forestière illégale [dans le Bassin du Congo].

- Le Forum des Nations Unies sur les forêts a tenu sa dixième session à Istanbul jusqu'au 19 avril ; le représentant de la République démocratique du Congo (RDC) y a fait le plaidoyer $d^{\prime}$ '« un des plus grands puits de carbone au monde». Selon lui, les arbres des forêts congolaises renferment 140 gigatonnes de carbone, faisant de la RDC I'un des plus grands puits de carbone au monde.

- Le 09 avril 2013, le FNUF a clos son débat ministériel par des déclarations appelant à une plus juste évaluation des contributions non monétaires « inestimables » des forêts : «On ne comprend pas toujours les enjeux, ce qui rend difficile la prise de décision » a reconnu M. Peter Holmgren, présent à ces assises.

- A l'occasion des cérémonies marquant le 20e anniversaire du CIFOR, organisées à Yaoundé les 22 et 23 mai prochains, I'IRD propose de faire le point sur les recherches menées en Afrique centrale, dans le cadre du Programme pilote régional (PPR FTH-AC).

- $\quad$ La superficie totale de forêts certifiées FSC (Forest Stewardship Council) au Cameroun vient de dépasser 1 Million d'hectares avec l'obtention de nouvelles certifications de trois unités forestières d'aménagement (UFA) gérées par la Société forestière et industrielle de la Doumé (SFID), une filiale Rougier et membre du Global Forest \& Trade Network (GFTN). Les trois UFA, couvrant 285667 ha, sont situées dans la région de l'Est du Cameroun, foyer des communautés Baka, considérées comme parmi les plus anciens habitants des forêts tropicales du Cameroun.

\section{AGENDA - CARNET}

- Au rythme des saisons : émission mensuelle qui se tient tous les premiers mercredis du mois, de 18 h05 à 18h55, Radio nationale, CRTV (Cameroun) (disponible sur ligne : http:/cifor. org/cobam/multimedia/radio.html )

- Atelier Régional de validation technique des Guides de Planification et d'Aménagement des Territoires forestiers en Afrique Centrale, 7-8 Mai 2013, Douala, Cameroun

- Atelier sur l'information, la sensibilisation, le rôle et la place des Parlementaires dans le processus REDD+ en Afrique Centrale, 7-9 Mai 2013, Ebolowa, Cameroun

- Conférence régionale sur le thème «Tendance sur la déforestation dans le bassin du Congo : Comment réconcilier la croissance économique et la protection des forêts », 16-17 Mai 2013, Kinshasa, RDC

- Atelier sur la Conservation des grands singes, 21-24 Mai 2013, Brazzaville, Congo

- Journée internationale de la Diversité biologique 2013 sous le thème : « Eau et biodiversité » 22 Mai 2013

- Conférence du CIFOR «sustainable management forest in Central Africa»: 22-23 mai 2013, hôtel Hilton Yaoundé, Cameroun

- Journée International de L'Environnement, 5 Juin 2013

- Journée Internationale de la Lutte contre la Désertification, 17 Juin 2013Fifth Africa Carbon Forum, 3-5 July 2013, Abidjan, Côte d'Ivoire

- $\quad$ Forum on Wetlands for Livelihoods, 8-12 July 2013, Kigali, Rwanda

- $\quad$ 19ème Session de la Commission des forêts et de la faune sauvage pour l'Afrique (CFFA) 30 Septembre - 4 Octobre 2013, Windhoek, Namibie

- UNCCD COP 11, 16-27 September 2013, Windhoek, Namibia

- World Food Day: Sustainable Food Systems for Food Security and Nutrition, 16 October 2013

- $\quad$ 49th Session of the International Tropical Timber Council (ITTC), 25-30 November 2013, Libreville, Gabon

- $\quad 5$ th Biennial C40 Mayors Summit (C40 Cities Climate Leadership Group (C40)), 4-6 February 2014, Johannesburg, South Africa 


\section{L'équipe de COBAM News}

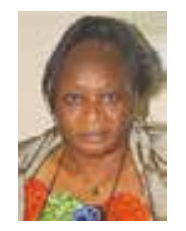

\section{Anne Marie Tiani}

Ecologiste, spécialisée dans le développement des approches participatives et les questions du genre. Elle est la coordonnatrice du projet COBAM.

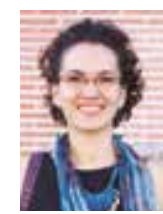

\section{Alba Saray Pérez Terán}

Spécialiste de suivi et évaluation participative pour le projet COBAM, a travaillé dans des projets d'environnement et changement climatique au Kenya, Sénégal, Thaïlande et Vietnam.

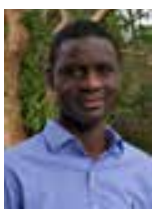

\section{Babatope Akinwande}

Babatope Akinwande est le Communicateur du CIFOR pour I'Afrique. Basé à Yaoundé, au Cameroun, il est chargé de développer et de mettre en œuvre la stratégie de communication pour l'Afrique du CIFOR.

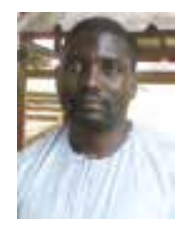

\section{Bérenger Tchatchou}

Ingénieur en Statistiques, avec une spécialisation en Economie appliquée. Il est en charge de la recherche socio-économique dans le cadre de la mise en œuvre des projets pilotes COBAM.

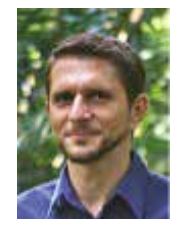

\section{Bruno Locatelli}

Docteur en sciences de l'environnement, mène des recherches sur les forêts et l'adaptation au changement climatique avec le CIRAD et le CIFOR, et est basé en Indonésie.

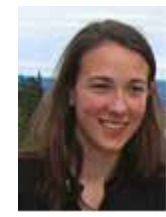

\section{Charlotte Pavageau}

Charlotte Pavageau est diplômée de l'Ecole du Génie rural, des Eaux et des Forêts (Agroparistech - ENGREF, Paris, France) et de l'École polytechnique (France). Elle coordonne I'analyse des politiques nationales liées aux forêts et au changement climatique.

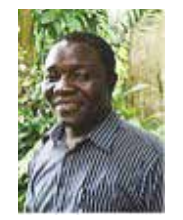

\section{Denis Sonwa}

Ecologiste, Dr Denis Sonwa est un chercheur du programme Forêts et Environnement du CIFOR et responsable du suivi scientifique du projet COBAM. II a coordonné CoFCCA, I'un des premiers projets sur les forêts et l'adaptation aux changements climatiques en Afrique centrale

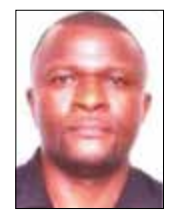

Eugene Loh Chia

Chercheur spécialiste des institutions dans le projet COBAM, en charge des aspects institutionnels et de gouvernance. Il a un Master en Etudes Internationales de l'Environnement de I'Université Norvégienne des Sciences de la Vie.

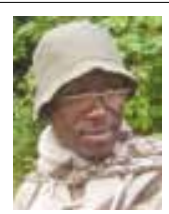

\section{Félicien Kengoum}

Chercheur dans le programme sur les forêts et la gouvernance du CIFOR et analyses des politiques forestières en Afrique centrale dans leurs rapports avec l'atténuation et l'adaptation au changement climatique.

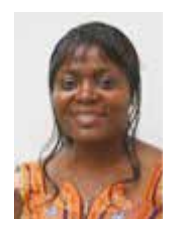

\section{Flore Ndong}

Chargée du suivi administratif et financier de toutes les activités liées au projet COBAM. Elle capitalise de plus de 9 ans de service dans plusieurs sociétés et organisations, en qualité d'assistante de direction et d'assistante administrative et financière.

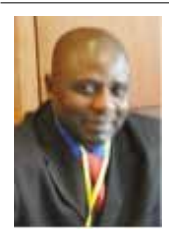

\section{Louis Bernard Cheteu}

Ingénieur forestier, Master of Philosophy en Environnement ; plus d'une dizaine d'années d'expérience dans la mise en œuvre des projets sous régionaux dans le secteur forêt environnement. Il est actuellement chargé du suivi et de l'accompagnement des projets pilotes COBAM.

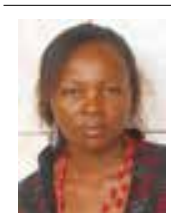

\section{Merline Touko}

Consultante en communication pour le projet COBAM-CIFOR. Elle s'occupe de la création audiovisuelle, de la réalisation et la diffusion de l'émission radiophonique "Au Rythme des Saisons"

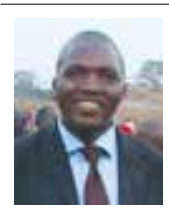

\section{Richard Sufo Kankeu}

Chercheur/Spécialiste SIG chargé du développement des méthodologies d'évaluation du stock carbone à travers les systèmes MNV, les SIG et la télédétection.

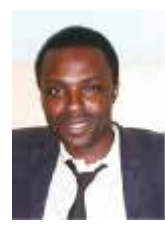

\section{Eric Essono Tsimi}

Consultant en communication, Eric Essono Tsimi est un journaliste et écrivain, qui s'occupe, au sein du COBAM, de l'édition et de la réalisation de la newsletter. Il est diplômé de l'Université Laval au Canada et de I'Université catholique d'Afrique centrale.

Photos par Anne Marie Tiani, Olivier Girard, Merline Touko, Sukaina Bharwani, Félicien Kengoum

COBAM est mis en œuvre par le Centre pour la recherche forestière internationale (CIFOR), dans le cadre du support de la Banque africaine de développement $(B A D)$ à la Communauté économique des Etats de l'Afrique centrale (CEEAC), pour financer le Programme de soutien à la conservation des écosystèmes du Bassin du Congo (PACEBCo).
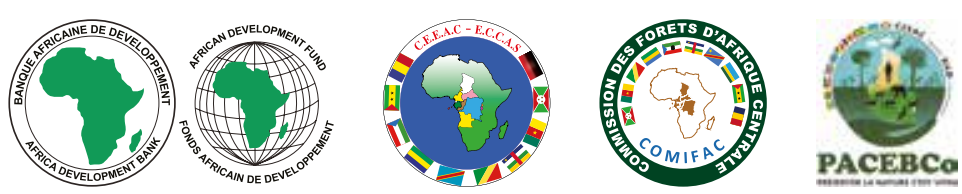

cifor.org/cobam

blog.cifor.org

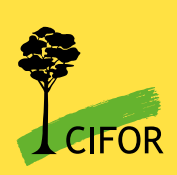

Center for International Forestry Research

CIFOR défend le bien-être humain, la conservation de l'environnement et l'équité en menant une recherche pour éclairer les politiques et les pratiques qui affectent les forêts dans les pays en développement. CIFOR est un centre de recherche du Consortium du CGIAR. Le siège du CIFOR est situé à Bogor, en Indonésie. CIFOR a également des bureaux en Asie, en Afrique et en Amérique du Sud. 